\title{
AVALIAÇÃO DO EFEITO DO FUNDEB SOBRE A PROFICIÊNCIA EM MATEMÁTICA DOS ALUNOS DO ENSINO MÉDIO NO ESTADO DO AMAZONAS
}

\section{EVALUATION OF THE EFFECT OF FUNDEB ON THE PROFICIENCY IN MATHEMATICS OF STUDENTS OF MIDDLE SCHOOL IN THE STATE OF AMAZONAS}

\author{
Felipe Diniz Leite ${ }^{1}$ \\ Rubicleis Gomes da Silva² \\ lara Maiara da Silveira ${ }^{3}$ \\ Rennan Biths de Lima Lima ${ }^{4}$
}

\begin{abstract}
RESUMO: O Fundo de Manutenção e Desenvolvimento da Educação Básica (FUNDEB) criado em 2006 é o maior mantenedor do sistema público educacional brasileiro, cujo objetivo principal é redistribuir os recursos vinculados à educação garantindo uma educação de qualidade. Nesse sentido, este trabalho buscou avaliar qual o impacto do FUNDEB sobre o nível de proficiência dos alunos da rede pública do estado do Amazonas para disciplina de matemática. Para tanto, o método utilizado foi o Diferenças em Diferenças. Os resultados mostram que o FUNDEB não apresenta efeitos sobre a proficiência dos alunos na disciplina avaliada. Entretanto, a análise dos seus efeitos nos quartis superior e inferior apresentou significância porém com impacto de dimensão negativo. Além disso, variáveis relacionadas às características dos alunos, professores e da escola influenciam tanto o desempenho do aluno quanto da escola.
\end{abstract}

Palavras- chave: FUNDEB, Diferenças em Diferenças e Avaliação de Impacto.

ABSTRACT: The Fund for the Maintenance and Development of Basic Education (FUNDEB) created in 2006 is the largest maintainer of the Brazilian public educational system, whose main objective is to redistribute resources linked to education, guaranteeing a quality education. In this sense, this work sought to evaluate the impact of FUNDEB on the level of proficiency of students in the public network of the state of Amazonas for mathematics discipline. For that, the method used was Differences in Differences. The results show that FUNDEB has no effect on students' proficiency in the subject. However, the analysis of its effects in the upper and lower quartiles presented significant but negative impact. In addition, variables related to the characteristics of students, teachers and the school influence both student and school performance.

KEYWORDS: Fund for the Maintenance and Development of Basic Education (FUNDEB), Differences in Differences and Evaluation of Impact.

\footnotetext{
${ }^{1}$ Bacharel em Ciências Econômicas - UFAC. Pós-Graduado em Economia Regional e Políticas Públicas - UFAC. Técnico da SUFRAMA.

${ }^{2}$ Professor de métodos quantitativos aplicados à economia - UFAC. Pesquisador Visitante EESPFGV.

${ }^{3}$ Bacharel em Ciências Econômicas - UFAC. Pós-Graduado em Economia Regional e Políticas Públicas - UFAC.

${ }^{4}$ Bacharel em Ciências Econômicas - UFAC. Mestre em Economia Aplicada - UFV. Doutoranda em economia - USP/ESALQ.
}

Revista de Estudos Sociais | Ano 2017, Pag. 1 


\section{INTRODUÇÃO}

\subsection{Considerações iniciais}

As políticas de financiamento da educação vêm sendo constantemente alvo de vários estudos principalmente em decorrência dos efeitos que estas proporcionam na qualidade de ensino. Desse modo, há um leque de teorias que mostram justamente o relacionamento entre o crescimento econômico e o aumento da mão de obra com os investimentos feitos na educação. Conforme Schultz (1973), quando se investe em educação se tem como resultado a formação do capital humano, o qual apesar de não poder ser vendido é um item que aumenta a produtividade econômica do fator trabalho.

No Brasil este tema vem sendo fruto de discussões desde a primeira constituição de 1824. Naquela época houve um grande debate para definir o financiamento e as responsabilidades pela oferta deste bem fundamental. As discussões a respeito deste tema perpassam por todo o processo constitucional brasileiro e perduram até os dias atuais, tendo como fato marcante a criação do Fundo Nacional de Desenvolvimento da Educação Básica (FUNDEB) no de 2006.

O FUNDEB é uma melhoria de seu antecessor, o Fundo de Manutenção e Desenvolvimento do Ensino Fundamental e de Valorização do Magistério (FUNDEF), o qual foi implantado no ano de 1998. A grande inovação do FUNDEF foi à estruturação do financiamento do ensino fundamental público no Brasil, que passou a acontecer de forma automática através da subvinculação de uma parcela dos recursos para esse nível de ensino. O grande problema deste Fundo e que foi sanado com o FUNDEB era a limitação à apenas o ensino fundamental receber tais recursos.

O principal objetivo do FUNDEB é promover a redistribuição dos recursos vinculados à educação. A ele foi passado o dever de assegurar a qualidade da educação básica no Brasil. Com vigência estabelecida de 2007 a 2020, o intuito é diminuir as desigualdades sociais e econômicas existentes nas diversas regiões do país, que tanto afetam o desenvolvimento da educação.

Os recursos do FUNDEB são compostos da seguinte forma: dos Fundos de participações dos Estados e Municípios, dos impostos (ICMS, IPlexp, ITCMD, IPVA, ITRm), dos recursos relativos as desonerações LC no87/96, além da complementação financeira por parte da União, a fim de completar o valor mínimo nacional por aluno. Tais recursos são destinados na sua grande maioria ao pagamento dos professores, especializações e demais gastos com pessoal, mas também à manutenção e desenvolvimento do ensino, influenciando os investimentos ligados à infraestrutura escolar.

O sistema educacional brasileiro apresenta sua estrutura descentralizada e marcada por discrepâncias regionais, principalmente nas regiões Norte e Nordeste, as quais apresentam os menores níveis de desenvolvimento econômico e social do país. Desse modo, esta pesquisa busca realizar uma avaliação da efetividade dessa política no estado do Amazonas, tendo em vista que este apresenta aproximadamente 3,8 milhões de habitantes, dos quais apenas 1,3 milhão (37\%) da população faz parte do sistema educacional amazonense. (IBGE, 2013). Em 2011 havia $11,66 \%$ dos amazonenses com mais de 5 anos sem saber ler e escrever. Enquanto em 2014, conforme dados da 
Pesquisa Nacional por Amostra e Domicílios (PNAD, 2015), essa taxa diminuiu para $9,16 \%$.

Apesar do Amazonas ter apresentado um bom desempenho no Índice de Desenvolvimento da Educação Básica (IDEB) de 2015, apresentando uma nota de 3,7 para os alunos do ensino médio, maior que sua média estabelecida de 3,3, o estado ainda possui dados preocupantes, bem como a região norte por inteiro, em relação a educação básica. Certamente todas as dificuldades vividas nessa região devem ser levadas em conta, como por exemplo, as grandes distâncias, o isolamento de muitas cidades e demais fatores típicos da região amazônica.

De modo geral, o sistema educacional brasileiro é problemático. Os índices de evasão e repetência são altíssimos e os alunos apresentam rendimentos aquém do desejado nas disciplinas de matemática e português. Nesse estudo, utilizou-se como base a disciplina de matemática, a qual é considerada uma matéria importante para o desenvolvimento das capacidades e habilidades da vida humana. Quanto à educação, ela mostra uma possibilidade de criar instrumentos que auxiliarão no aprimoramento do raciocínio lógico, da inventividade e a capacidade criadora do indivíduo. (LEONARDO et. al, 2014).

Diante do exposto, é relevante responder a seguinte pergunta: Quais os impactos do FUNDEB na proficiência em matemática dos alunos do ensino médio da educação pública amazonense?

Conforme a literatura, uma boa proxy para mensurar a qualidade do ensino é utilizar os testes de proficiência de capacidade cognitiva do aluno. (SILVEIRA, 2014). Portanto, utilizou-se aqui, os resultados do Sistema de Avaliação da Educação Básica (SAEB) para a disciplina de matemática como parâmetro de avaliação da qualidade do ensino nas escolas do Amazonas.

Segundo o Instituto Nacional de Estudos e Pesquisas Educacionais Anísio Teixeira (INEP, 2011), a pontuação para tal disciplina vai de 0 a 425 pontos, sendo que o nível considerado adequado no desempenho dos alunos é de 350 pontos respectivamente, conforme a nota obtida pelo Sistema de Avaliação da Educação Básica (SAEB).

O SAEB tem como objetivo criar indicadores para monitorar o processo de ensino-aprendizagem, com o intuito de melhorar a qualidade de ensino, principalmente das disciplinas consideradas como pilares para as demais, isto é, língua portuguesa e matemática. Os participantes do SAEB são alunos que frequentam a $4^{\mathrm{a}}$ e $8^{\mathrm{a}}$ séries do ensino fundamental e $03^{\circ}$ série do ensino médio. (DOURADO, 2005).

A importância deste trabalho é verificar se a aplicação dos recursos do FUNDEB apresentam resultados positivos ou negativos no desempenho dos alunos, além de construir um diagnóstico do sistema educacional do Amazonas, possibilitando a identificação de potencialidades ou fragilidades das políticas públicas em educação, e assim, auxiliar os tomadores de decisão e formuladores de políticas no que diz respeito a melhoria dos serviços educacionais no estado do Amazonas.

O objetivo geral deste estudo é avaliar o impacto do FUNDEB sobre o nível de proficiência na disciplina de matemática dos alunos da rede pública e como isso demonstra a qualidade do ensino no estado do Amazonas. Especificamente, pretende-se: a) Traçar o perfil das escolas do ensino médio do 
estado do Amazonas; b) Mostrar o efeito do FUNDEB sobre a proficiência em matemática.

Para isso, parte-se da hipótese de que o maior investimento da educação básica não resultará em um maior desempenho educacional dos alunos do ensino médio na rede pública amazonense.

Nos últimos anos, várias estudos foram realizados para investigar o desempenho das políticas educacionais, bem como a qualidade da educação. Hanushek (2002) avaliou como as políticas de gastos educacionais estão relacionadas com a aquisição de insumos escolares, sendo os professores um dos principais insumos no processo de aprendizagem. Menezes-Filho (2007) fez um estudo para verificar quais fatores determinam o desempenho escolar 0 Brasil. Seus resultados mostraram que há um efeito escola atuando no desempenho dos alunos da rede pública, isto é, existem diferenças entre as escolas em decorrência de diferentes investimentos em insumos escolares. Além disso, Silveira (2014) analisou o impacto do FUNDEB sobre o desempenho dos alunos do ensino médio nas escolas brasileiras e detectou que essa política melhora a qualidade da educação, entretanto, as escolas com pequeno desempenho dos alunos pouco foram influenciadas para equidade nas escolas de ensino médio.

O presente trabalho está dividido, além desta introdução, em mais quatro seções. Na segunda seção é feita uma abordagem do referencial teórico que substanciou a análise do trabalho. O referencial analítico é discutido na terceira seção. Em seguida, são apresentados, na quarta seção, os resultados e discussões, e por fim, na última seção é apresentada a conclusão do trabalho.

\section{A importância da Matemática e a realidade da proficiência no Amazonas}

De maneira geral, o sistema educacional de ensino brasileiro vem ganhando destaque em estudos nacionais, não apenas pela democratização do acesso à educação, mas também pelo fato da escola possuir o papel de educador das novas gerações, uma vez que os anos iniciais de escolarização são primordiais no sucesso e aprendizado do aluno já que as crianças se encontram no momento ideal para o desenvolvimento das habilidades cognitivas.

Ademais, como destaca Silveira (2014), a educação é um fator crucial no processo de desenvolvimento econômico de um país. Assim, é relevante não só seu acesso, mas primordialmente sua qualidade. Nesse cenário surgem as avaliações externas que mensuram a qualidade do ensino. $O$ fato é que o Brasil não vem apresentando o desempenho desejado nessas avaliações, que servem como instrumentos para elaboração de políticas públicas educacionais.

A maioria dos estudos que mensuram a qualidade do ensino apontam que a disciplina de matemática apresenta uma proficiência aquém do recomendado. Historicamente, esta disciplina é difundida como sendo de difícil compreensão e detentora de elevados índices de reprovação no Brasil. Assim, alunos que terminam o ensino médio, em escolas públicas brasileiras, de maneira geral, não conseguem resolver problemas com operações básicas, como equações de primeiro grau. (SEDEAM, 2015).

Conforme preceitua Santos (2006), esta ciência subsidia à ciência moderna, não só o instrumento de análise, mas também a lógica investigativa e Revista de Estudos Sociais | Ano 2017, Pag. 4 
a própria estrutura da matéria. O Ministério da Educação (MEC, 1997) através dos seus parâmetros curriculares nacionais enuncia que a matemática ajuda a formar o pensamento e o raciocínio dedutivo. No campo formativo ela contribui para criar no aluno uma capacidade para resolutiva de problemas cotidianos, além de prepara-los para analisar e enfrentar situações novas.

Nesse sentido a Matemática busca ajudar o indivíduo a participar do processo de construção de conhecimento objetivando resoluções de questionamentos do dia a dia. O Brasil é um dos últimos colocados no ranking mundial nas competências nesta disciplina. Conforme Fernandes (2016) os dados da Organização para a Cooperação e Desenvolvimento Econômico mostram o quão atrasados estão os alunos brasileiros que ficaram em 58임 luar dentre os 65 países que participaram desta avaliação.

Conforme Toledo (2016) há uma disparidade no aprendizado dessa disciplina entre as escolas públicas e privadas e o estado do Amazonas figura no topo da lista, é o estado com maior desigualdade no país, onde segundo a metodologia adota pelo instituto aponta uma diferença de 37,70 pontos entre as duas redes de ensino.

Desse modo, os dados do SAEB, para os anos de 2005 e 2011, apontam que a média nacional no desempenho na disciplina de matemática foi de 260 pontos em 2005 e 265 pontos no ano de 2011. Neste período, o estado do Amazonas obteve uma média acima da nacional com 281,83 em 2005 e 276,3 em 2011, ficando ainda bem abaixo do considerado adequado que é 350 pontos.

Conforme dados do INEP (2013), ao final do último ano do ensino fundamental, quase metade dos alunos das escolas públicas brasileiras, $89 \%$, encontram-se abaixo do nível de proficiência em matemática desejado. Na rede privada, o cenário não é muito diferente. O mesmo cenário pode ser visualizado para o estado do Amazonas, onde ainda conforme dados do INEP, 92\% dos alunos que estão terminando o ensino fundamental estão abaixo do nível desejado de proficiência.

Os dados são preocupantes, pois é possível visualizar que isso irá se repercutir também no ensino médio. Em 2009, a proficiência dos alunos foi menor do que em 1995 - 265,5 e 272,1, respectivamente. Algo acontece com o ensino desta disciplina no país, talvez os caminhos adotados não estão compatíveis com o mundo real do aluno. Entretanto, é preciso verificar como a disciplina vem sendo ministrada nas salas de aula e como os professores estão sendo preparados para seu ensino.

Estudos mostram que práticas como passar lição de casa em todas ou quase todas as aulas, corrigi-la, sempre explicar a matéria até que todos os alunos entendam e que possam relacionar os conteúdos da disciplina às situações cotidianas e propor resolução de problemas influenciam positivamente no desempenho dos estudantes e ainda mais quando combinadas.

\section{REFERENCIAL ANALÍTICO}

\subsection{Método de Diferenças em Diferenças}

Esta é uma metodologia bastante usual quando se fala em avaliação de políticas públicas e programas sociais, o método permite fazer um isolamento de interesse através da comparação com um determinado grupo de controle. Assim a amostra fica dividida em um grupo de controle antes da mudança, um grupo Revista de Estudos Sociais | Ano 2017, Pag. 5 
de controle depois da mudança e por fim, um grupo de tratamento antes e depois da mudança. Para Foguel (2012), o maior problema é determinar o grupo contrafactual dos tratados, é necessário minimizar o viés de seleção utilizando procedimentos e hipóteses para tais fins.

Foguel (2012) salienta que a regressão diff-in-diff possui hipóteses e uma metodologia que pode eliminar esse viés. Para ele este método é enquadrado como um método não experimental, pois os grupos de tratamento e controle são definidos por forças naturais ou econômicas. O método de diferenças em diferenças tem como fundamento uma dupla subtração como pode ser observado no Quadro 1.

Quadro 1 - Resumo do Método de diferenças em diferenças.

\begin{tabular}{cccc}
\hline & Antes & Depois & Diferenças \\
\hline Controle & A & B & $A-B$ \\
Tratamento & C & D & C - D \\
Diferenças & $A-C$ & $B-D$ & $(A-C)-(B-D)$ \\
& & & ou \\
& & & $(A-B)-(C-D)$ \\
\hline
\end{tabular}

Fonte: Centro de Políticas Sociais da Fundação Getúlio Vargas

Pode-se descrever o grupo a ser estudado da seguinte forma $G_{i}\left(G_{i}\right.$ $E\{0,1\})$, o qual $G_{i}=1$ é o indivíduo ou grupo que está recebendo o tratamento, e $G_{i}=0$ são os indivíduos ou grupos de controle, aqueles que não recebem o efeito da política pública. Quanto ao tempo, assume que $T_{i}\left(T_{i} E\{0,1\}\right)$ onde $T_{i}=$ 0 e $T_{i}=1$ são os momentos antes e depois da implantação do programa ou política pública respectivamente. A ideia é que se tenha os resultados e seus determinantes antes e depois da implementação do programa, tanto para o grupo de controle quanto para seus determinados contrafactuais. É importante salientar que ambos os grupos devem seguir uma mesma tendência.

Conforme Foguel (2012), encontrar um contrafactual que represente de forma significativa a situação de não tratado é, dentre outros, um dos desafios na análise de impacto, pois não é possível encontrar as duas situações (tratamento e controle) no mesmo indivíduo ou grupo simultaneamente.

Neste trabalho se usa a metodologia de Diferenças em Diferenças para avaliar os impactos do FUNDEB sobre o desempenho médio dos alunos. Em $T_{i}$ igual a 0 se tem as escolas observadas em 2005, o qual indica um período anterior ao programa. Já em $T_{i}$ igual a 1 representa o as escolas observadas em 2011, período posterior a implantação do FUNDEB. O procedimento adotado neste trabalho segue o mesmo de Menezes-Filho e Pazzelo (2007), Franco (2008) e Silveira (2014), os quais utilizaram como grupo de controle as escolas privadas e como grupo de tratamento a rede pública que é abrangida pelo FUNDEB. Há autores que dizem não ser ideal o uso de escolas particulares como contrafactual, mas se pode justificar o seu uso, como salienta Foguel (2012), pelo fato de que o grupo de controle deve apresentar a mesma tendência em relação ao desempenho escolar.

Partindo deste pressuposto temos como resultado $Y_{i}$ em que $Y_{1 i}$ é o desempenho médio da escola $i$, a qual pertence ao grupo de tratamento, e $Y_{0 i}$ é o desempenho médio da escola $i$, que pertence ao grupo de controle. Então, o resultado observado para a escola $i$ é dado por:

Revista de Estudos Sociais | Ano 2017, Pag. 6 


$$
Y_{i}=Y_{0 i}\left(1-I_{i}\right)+Y_{1 i} I_{i}
$$

Em que $Y_{i}$ é a proficiência média dos alunos que pertencem a escola $i$ na disciplina de matemática e $I_{i}=G_{i} T_{i}$ é o status de tratamento da escola $i$.

Definindo que grupo de controle e tratamento segue a mesma tendência no desempenho escolar, é possível identificar o efeito causal da variável de interesse. $O$ resultado para a escola $i$ na ausência da intervenção satisfaz a equação:

$$
Y_{0 i t}=\alpha+\beta T_{i}+\eta G_{i}+\varepsilon_{i t}
$$

Onde $\beta$ é o efeito de tempo comum a todas as escolas, $\eta$ é um efeito específico de grupo que não varia no tempo, e $\varepsilon_{i t}$ seja i.i.d. entre tempo e indivíduos. As unidades de observação devem apresentar uma mesma composição entre os períodos anterior e posterior à implantação do programa. Com essa pressuposição, o efeito de tratamento deve ser constante entre os grupos:

$$
Y_{1 i t}-Y_{0 i t}=\tau
$$

Combinando as equações (9) e (10) $Y_{i}$ pode ser escrito da seguinte forma:

$$
Y_{i t}=\alpha+\beta T_{i}+\eta G_{i}+\tau I_{i}+\varepsilon_{i t}
$$

Esta expressão é a equação básica do método de Diferenças em Diferenças onde $\tau$ indica o efeito de tratamento do programa. Este parâmetro também pode ser estimado através de MQO - Métodos Quadrados Ordinários, dizendo que $G(0)$ são as escolas privadas que pertencem ao grupo de controle e $G(1)$ as escolas de tratamento, ou seja, as escolas da rede pública abrangidas pelo FUNDEB, o estimador de $\tau$ por MQO pode ser expresso como:

$$
\tau_{M Q O}=(\bar{y} G(1) 0)-(\bar{y} G(0) 1-\bar{y} G(0) 0)
$$

É possível ainda no método de Diferenças em Diferenças incluir variáveis de controle adicionais na equação de estimação (4). A importância desta inclusão esta no fato de que essas variáveis controlam possíveis diferenças que ocorram em diferentes períodos de tempo, fazendo com que não se confunda com os efeitos de tratamento. Além disso são incluídas algumas variáveis dummies, as quais captam diferenças de efeito do programa dentre os grupos. A equação (11) ajustada por um vetor de características observáveis $X_{i t}$ fica escrita como:

$$
Y_{i t}=\alpha+\beta T_{i}+\eta G_{i}+\tau I_{i}+\gamma X_{\ddot{i}}+\varepsilon_{i t}
$$

Em que $Y_{i t}$ é a proficiência média da escola na disciplina de matemática; $T_{i}$ é uma variável dummy de tempo que recebe valor igual a 0 para o período antes do FUNDEB e valor igual a 1 para período posterior a implantação do programa; $G_{i}$ é uma dummy que se refere a qual o grupo pertence a escola, Revista de Estudos Sociais | Ano 2017, Pag. 7 
sendo o valor igual a 1 para as escolas de tratamento e valor igual 0 para as escolas de controle; $l_{i}$ indica o status do tratamento, é a interação entre as dummies de tempo e grupo; $X_{i t}$ é um vetor de características médias dos alunos e da escola, bem como da família.

O vetor $X_{i t}$ apresenta importantes variáveis de controle, as quais segundo a literatura são importantes fatores na explicação do desempenho dos alunos. Foram incluídas as variáveis dummies que indicam cor dos alunos, alunos que iniciaram os estudos na pré-escola e alunos que trabalham fora de casa; variáveis dummies para as escolas que apresentam bibliotecas, computadores com acesso à internet e elevado índice de absenteísmo dos professores; e dummies voltadas aos professores, como por exemplo: anos de experiências, salários e escolaridade dos professores.

\subsection{Fonte de dados}

Os dados utilizados neste artigo foram elaborados pelo Sistema de Avaliação da Educação Básica - SAEB. É desenvolvido pelo Instituto Nacional de Estudos e Pesquisas Educacionais Anísio Teixeira - INEP, por meio de amostragem das redes pública e privada nos estados e Distrito Federal. A aplicação acontece a cada dois anos para os alunos da $5^{\underline{a}}$ e $9^{\underline{a}}$ séries do ensino fundamental e $3^{a}$ série do ensino médio, os alunos são avaliados nas disciplinas de matemática e português. Segundo o INEP, a amostragem usada pelo SAEB são aleatórias, probabilísticas e representativas da população de referência, é utilizada uma metodologia que garante a precisão nas estimativas.

Os dados usados neste trabalho são das edições de 2005 e 2011 do SAEB, os quais se referem às escolas da rede pública e privada. A proficiência escolar analisada tem escala que varia de 0 a 500, sendo 0 a nota mínima obtida no teste e 500 a nota máxima. Segundo o MEC o nível adequado para os alunos na $3^{\text {a }}$ série do ensino médio na disciplina de matemática é de 350 pontos.

Abaixo na Tabela 1 é descrito todas as variáveis que foram utilizadas no processo de estimação do impacto do FUNDEB na proficiência de matemática dos alunos.

Tabela 1 - Descrição das variáveis relativas às características dos alunos, professores e escolas.

\begin{tabular}{|c|c|}
\hline Variáveis & Descrição \\
\hline Profic_Mat & Proficiência média da escola em matemática \\
\hline Rede_ensino & Dummy de rede de ensino (pública $=1$, privada $=0$ ) \\
\hline Tempo & $\begin{array}{l}\text { Dummy de tempo (depois do programa }=1 \text {, antes do } \\
\text { programa }=0 \text { ) }\end{array}$ \\
\hline Efeito_Fundeb & $\begin{array}{l}\text { Dummy resultante da operação: FUNDEB * Tempo (escola } \\
\text { pública após ser beneficiada pelo FUNDEB }=1 \text {, escola não } \\
\text { beneficiada pelo FUNDEB }=0 \text { ) }\end{array}$ \\
\hline Alun_sexo_masc & Proporção de alunos do sexo masculino \\
\hline Alun_cor_preta & Proporção de alunos da cor preta \\
\hline Alun_trab & Proporção de alunos que trabalham fora de casa \\
\hline
\end{tabular}

Revista de Estudos Sociais | Ano 2017, Pag. 8 


\begin{tabular}{|c|c|}
\hline Alun_reprov & $\begin{array}{l}\text { Proporção de alunos que foram reprovados } 1 \text { ou mais } \\
\text { vezes }\end{array}$ \\
\hline Alun_freq_pre & Proporção de alunos que frequentaram a pré-escola \\
\hline Alun_pai_sup & Proporção de alunos que \\
\hline Prof & $\begin{array}{l}\text { Proporção de professores que recebem até } 3 \text { salários } \\
\text { mínimos }\end{array}$ \\
\hline Prof_exp_5 & Proporção de professores com experiência de até 5 anos \\
\hline Prof_elev_absent & $\begin{array}{l}\text { Dummy de absenteísmo: a escola com elevado } \\
\text { absenteísmo de professores? }(\operatorname{sim}=1 \text {, não }=0)\end{array}$ \\
\hline Esc_biblioteca & $\begin{array}{l}\text { Dummy de biblioteca: a escola possui biblioteca? }(\operatorname{sim}=1 \text {, } \\
\text { não }=0) \\
\text { Dummy de acesso a internet: a escola tem computadores }\end{array}$ \\
\hline Esc_c & $\begin{array}{l}\text { com acesso à internet para uso dos alunos? }(\operatorname{sim}=1 \text {, não } \\
=0)\end{array}$ \\
\hline
\end{tabular}

Fonte: Elaborado pelos autores

\section{RESULTADOS E DISCUSSÃO}

4.1. Perfil das escolas do ensino médio no estado do Amazonas

O presente estudo utilizou a Avaliação Nacional da Educação Básica (ANEB) como referência na avaliação do impacto do FUNDEB na proficiência em matemática dos alunos do Ensino Médio do estado do Amazonas. Esta avaliação é realizada no âmbito do SAEB, para os anos de 2005 e 2011.

A Tabela 2, mostra a média dos resultados obtidos pelos alunos na avaliação, nos anos de 2005 e 2011, isto é, antes e depois do FUNDEB. Observa-se pelo teste de média que a proficiência média na disciplina de matemática é diferente nas escolas da rede pública e privada. Sendo assim, observa-se que na avaliação de matemática realizada em 2005, a diferença entre as redes era de 69,67 pontos, das quais as escolas privadas apresentaram um melhor desempenho em relação a nota obtida pelas escolas públicas.

No ano de 2011, a avaliação registrou uma redução de aproximadamente 10 pontos em relação a 2005 , chegando a 59,26 pontos. Entretanto, apesar dessa queda, houve uma piora na qualidade de ensino de matemática no estado do Amazonas para ambas as redes. Ressalta-se ainda, que nos dois períodos analisados as escolas da rede pública e privada não atingiram a pontuação considerada adequada pelo INEP, que como já dito anteriormente é de 350 pontos.

Em contrapartida, os dados do SAEB, para os anos de 2005 e 2011, apontam que a média nacional no desempenho na disciplina de matemática foi de 260 pontos em 2005 e 265 pontos no ano de 2011. Neste período, o estado do Amazonas obteve uma média acima da nacional com 281,83 em 2005 e 276,3 em 2011, mostrando um decréscimo na proficiência mesmo após a implantação do FUNDEB. 
Tabela 2 - Média da proficiência em matemática e teste de média das escolas de ensino médio, privadas e públicas, 2005 e 2011.

\begin{tabular}{lrrrrr}
\hline \multirow{2}{*}{ Escolas } & \multicolumn{2}{c}{2005} & \multicolumn{2}{c}{2011} & \multirow{2}{*}{$\begin{array}{c}\text { Variação } \\
(\%)\end{array}$} \\
\cline { 2 - 5 } & Média & $\begin{array}{r}\text { DesvPa } \\
\text { d }\end{array}$ & Média & $\begin{array}{r}\text { DesvPa } \\
\text { d }\end{array}$ & \\
\hline Escolas da rede privada & 316,67 & 37,86 & 305,93 & 33,82 & $(3,40)$ \\
Escolas da rede pública & 247,00 & 35,13 & 246,67 & 32,76 & $(0,13)$ \\
Diferença entre as redes & 69,67 & - & 59,26 & - & $(14,94)$ \\
$\begin{array}{l}\text { P-valor do teste de } \\
\text { média }^{5}\end{array}$ & 0,0000 & & 0,0000 & & \\
\hline
\end{tabular}

Fonte: Resultado da pesquisa.

Nota: * Significativo a $1 \%$.

A Tabela 3 mostra o valor médio das variáveis que caracterizam o perfil das escolas amazonenses nos anos de 2005 e 2011. Com relação as características individuais dos alunos é possível notar através do teste de média, que as variáveis relacionadas a proporção de alunos do sexo masculino e a proporção de alunos que declararam ser da cor preta são estatisticamente iguais entre as duas redes de ensino, isto é, a quantidade de alunos da rede pública é igual a quantidade de alunos da rede privada para estas características.

Quanto a proporção de alunos que reprovaram em anos anteriores, verifica-se que a diferença entre as redes pública e privada é discrepante, onde $35 \%$ dos estudantes da rede pública já tiveram alguma reprovação no seu histórico escolar. Já para variável relacionada aos alunos que frequentaram a pré-escola, os dados mostram que o maior percentual está na rede privada, tanto em 2005 quanto em 2011. Além disso, a variável que apresenta maior discrepância é a escolaridade dos pais, em que apenas $6,51 \%$ dos pais dos alunos da rede pública possuíam ensino superior em 2005, enquanto em 2011 houve um aumento de aproximadamente 4 pontos percentuais (p.p.), chegando em $10 \%$ dos estudantes com pais possuindo tal nível de ensino.

Tabela 3 - Valor médio (\%) e teste de média das variáveis referente ao perfil dos alunos, professores e infraestrutura das escolas.

\begin{tabular}{|c|c|c|c|c|c|c|}
\hline \multirow[b]{2}{*}{ Variáveis } & \multicolumn{3}{|c|}{2005} & \multicolumn{3}{|c|}{2011} \\
\hline & $\begin{array}{l}\text { Escolas } \\
\text { Privada }\end{array}$ & $\begin{array}{l}\text { Escolas } \\
\text { Pública }\end{array}$ & $\begin{array}{l}\text { Teste }^{6} \\
\text { P-valor }\end{array}$ & $\begin{array}{l}\text { Escolas } \\
\text { Privada }\end{array}$ & $\begin{array}{l}\text { Escolas } \\
\text { Pública }\end{array}$ & $\begin{array}{r}\text { Teste } \\
\text { P-valor }\end{array}$ \\
\hline Alun_sexo_masc & 51,6106 & 45,1838 & $0,2518^{N S}$ & 47,6490 & $\begin{array}{r}45,437 \\
5\end{array}$ & $\begin{array}{r}0,5160^{N} \\
s\end{array}$ \\
\hline Alun_cor_preta & 4,5973 & 3,7426 & $0,6818^{\mathrm{NS}}$ & 5,3528 & 35,5400 & $0,8526^{N}$ \\
\hline
\end{tabular}

\footnotetext{
${ }^{5}$ Teste de diferença na média das variáveis nos períodos de referência, sob a hipótese nula $\left(\mathrm{H}_{0}\right)$ de médias iguais entres os grupos de tratamento (escolas públicas) e de controle (escolas privadas).

${ }^{6}$ Teste de diferença na média das variáveis nos períodos de referência, sob a hipótese nula $\left(\mathrm{H}_{0}\right)$ de médias

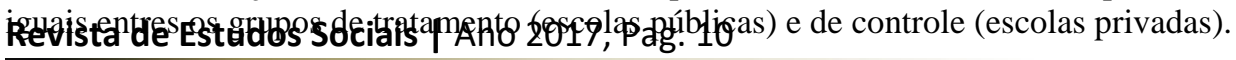




\begin{tabular}{|c|c|c|c|c|c|c|}
\hline Alun_trab & 21,9474 & 44,0392 & $0,0006^{*}$ & 19,0099 & $\begin{array}{r}37,929 \\
8\end{array}$ & $0,0000^{*}$ \\
\hline Alun_reprov & 17,7112 & 49,5756 & $0,0000^{*}$ & 16,7598 & $\begin{array}{r}40,488 \\
6\end{array}$ & $0,0000^{*}$ \\
\hline Alun_freq_pre & 62,7563 & 20,7445 & $0,0000^{*}$ & 47,2769 & $\begin{array}{r}43,424 \\
3\end{array}$ & $0,0000^{*}$ \\
\hline Alun_pai_sup & 38,4085 & 6,5194 & $0,0000^{*}$ & 42,6428 & 9,9661 & $0,0000^{*}$ \\
\hline Prof_sal_3sm & 28,5714 & 36,1111 & $0,5893^{N S}$ & 43,3333 & $\begin{array}{r}77,291 \\
6\end{array}$ & $0,0007^{\star}$ \\
\hline Prof_exp_5 & 16,0714 & 12,5000 & $0,6899^{N S}$ & 28,1250 & $\begin{array}{r}18,703 \\
7\end{array}$ & $\begin{array}{r}0,2133^{N} \\
S\end{array}$ \\
\hline Prof_elev_absent & 28,5714 & 41,1765 & $0,4820^{N S}$ & 12,5000 & $\begin{array}{r}40,000 \\
0\end{array}$ & $0,0080^{*}$ \\
\hline Esc_biblioteca & 100,0000 & 100,0000 & $0,5420^{N S}$ & 96,7742 & $\begin{array}{r}93,333 \\
3\end{array}$ & $\begin{array}{r}0,5156^{N} \\
S\end{array}$ \\
\hline Esc_comp_net & 85,7143 & 16,6667 & $0,0000^{*}$ & 96,7742 & $\begin{array}{r}82,222 \\
2 \\
\end{array}$ & $0,0547^{\star *}$ \\
\hline
\end{tabular}

Fonte: resultado da pesquisa.

Nota: ${ }^{*}$ Significativo a $1 \%,{ }^{* *}$ significativo a $5 \%,{ }^{* \star *}$ significativo a $10 \%$ e ${ }^{\text {NS }}$ não significativo.

Ainda de acordo com a Tabela 3, no que se refere às características dos professores, observa-se, conforme teste de média, que nenhuma característica avaliada apresentou diferenças entre as redes de ensino no ano de 2005. Já em 2011, observa-se que escolas da rede pública apresentam um maior nível de absenteísmo quando comparadas com as escolas privadas. Ademais, com relação à remuneração dos professores, pode-se verificar que a maioria dos professores recebem até 3 salários mínimos, tanto na rede privada quando na rede pública, mostrando conforme Silveira (2014) que este nível salarial torna a carreira de docência pouco atrativa.

No tocante as variáveis que caracterizam a infraestrutura das escolas, o teste de média demonstrou que a proporção de escolas com bibliotecas são estatisticamente iguais nas duas redes para os dois períodos. Com relação às escolas com computadores e acesso à internet, observa-se que esta variável foi estatisticamente significativa, mostrando que em 2005 a rede privada de ensino apresentava uma proporção de $85,71 \%$, enquanto na rede pública apenas $16,66 \%$ das escolas tinham computadores ligados à rede mundial. Entretanto, depois do FUNDEB, é possível verificar uma grande evolução desta variável para as escolas da rede pública, aumentando 65 p.p. em 2011, chegando a um percentual de $82,22 \%$ neste ano.

4.2. Efeito médio do FUNDEB sobre a proficiência dos alunos na disciplina de matemática das escolas amazonenses

Neste estudo foi utilizada a metodologia de Diferenças em Diferenças para verificar os efeitos do FUNDEB sobre a proficiência dos alunos na disciplina de matemática no estado do Amazonas, além do efeito que outras variáveis proporcionam ao nível de proficiência, como mostra a Tabela 4.

Revista de Estudos Sociais | Ano 2017, Pag. 11 
Os resultados apontam para um não efeito do FUNDEB sobre a proficiência dos alunos em matemática, como se verifica por meio da não significância da variável Efeito_Fundeb, que traduz a interação entre as variáveis FUNDEB e tempo, ou seja, antes e depois do programa, implicando assim, que o fato da escola ser beneficiária do Fundo não the assegura um melhor desempenho na disciplina de matemática. Sendo assim, a hipótese inicial está correta, pois o FUNDEB não foi determinante na melhoria do desempenho dos alunos no estado do Amazonas.

O FUNDEB é garantidor constitucional de recursos para a educação, isso significa que o dinheiro tem e deve ser aplicado. Salienta-se também que os índices de fraudes em relação ao fundo são muito baixos. Assim sendo, é importante fazer uma reflexão para a destinação do recurso, possivelmente ele está sendo empregado em varáveis que não melhoram o desempenho dos alunos. Talvez o problema não esteja no salário dos professores e nem apenas nas estruturas das escolas, possivelmente se esteja em meio a um grande ciclo vicioso que se inicia na pré-escola, passa pela formação no ensino regular e finda na formação acadêmica dos futuros professores.

Tabela 4 - Resultado da regressão do método Diferença em Diferença, estimada por mínimos quadrados ordinários, para a proficiência média em matemática dos alunos da $3^{\underline{a}}$ série do ensino médio do Amazonas.

\begin{tabular}{lcccc}
\hline Variáveis & Coeficiente & Erro Padrão & Estatística - t & P-valor \\
\hline Efeito_Fundeb & 1.492683 & 13.27152 & 0,1100 & $0,9110^{\mathrm{NS}}$ \\
Rede_ensino & -9.542743 & 12.52606 & $-0,7600$ & $0,448^{\mathrm{NS}}$ \\
Tempo & -10.31411 & 8.150444 & $-1,2700$ & $0,2090^{\mathrm{NS}}$ \\
Alun_sexo_masc & 47.90926 & 18.45147 & 2,6000 & $0,0110^{* *}$ \\
Alun_cor_preta & 63.23854 & 48.93999 & 1,2900 & $0,2000^{\mathrm{NS}}$ \\
Alun_trab & -57.64463 & 16.86215 & $-3,4200$ & $0,0010^{*}$ \\
Alun_reprov & -86.36833 & 16.24838 & $-5,3200$ & $0,0000^{*}$ \\
Alun_freq_pre & 17.53861 & 18.73329 & 0,9400 & $0,3520^{\mathrm{NS}}$ \\
Alun_pai_sup & 23.16563 & 18.44096 & 1,2600 & $0,2120^{\mathrm{NS}}$ \\
Msal_ate3 & -11.6695 & 6.355962 & $-1,8400$ & $0,0700^{* *}$ \\
Prof_exp_5a & -16.85328 & 8.286059 & $-2,0300$ & $0,0045^{*}$ \\
Prof_elev_absent & -4.595589 & 5.247043 & $-0,8800$ & $0,3840^{\mathrm{NS}}$ \\
Esc_biblioteca & 41.59681 & 24.02196 & 1,7300 & $0,0870^{* *}$ \\
Esc_comp_net & -1.463912 & 7.477315 & $-0,2000$ & $0,8450^{\mathrm{NS}}$ \\
Constante & 264.1006 & 29.45896 & 8,9700 & $0,0000^{*}$ \\
R & 0,7781 & & & \\
\hline RevistadeEstudos & & & &
\end{tabular}

Revista de Estudos Sociais | Ano 2017, Pag. 12 
Fonte: resultado da pesquisa

Nota: " Significativo a $1 \%,{ }^{* *}$ significativo a $5 \%,{ }^{* * *}$ significativo a $10 \%$ e NS Não Significativo.

As variáveis Alun_cor_preta, Alun_freq_pre, Alun_pai_sup, Prof_elev_absent e Esc_comp_net também não explicam o nível de proficiência em matemática dos alunos do ensino médio, pois não foram significativos estatisticamente.

É importante aqui fazer um destaque para a variável Esc_comp_net, pois como se propaga no meio de várias discussões, as quais justificam que é preciso colocar computador e internet em todas as escolas e que incluir a escola na era digital é imprescindível para melhorar a educação. O que fica claro na Tabela 4 é que o fato de a escola ter computador não é garantia de ensino de qualidade, aliás, os dados mostram que ela está influenciando negativamente na nota de proficiência. Logicamente que este estudo não é contra o computador, mas é preciso usá-lo para o bem do aluno, é preciso ter professores capacitados para usá-los da maneira mais eficiente e em prol do crescimento escolar.

Quanto às características dos alunos, a variável Alun_sexo_masc se mostrou significativo. Assim, a cada 1 ponto percentual (p.p) de aumento na proporção de alunos do sexo masculino, o desempenho da escola aumenta em 47,90 pontos, demostrando maior facilidade com a disciplina de matemática do que as mulheres.

Além disso, a cada aumento em 1 p,p. na proporção de alunos que exercem alguma atividade profissional, a escola perde 57,64 pontos no desempenho médio da proficiência em matemática e isso era o esperado já que como mostra Menezes-Filho (2011), os alunos que não precisam trabalhar para compor a renda bruta familiar, são mais aptos a discernir o conteúdo apresentado pelos professores. Além disso, Machado et. al. (2008), mostram que os alunos do ensino médio que trabalham fora de casa apresentam uma participação reduzida nas atividades escolares, impactando negativamente no seu rendimento.

Similarmente, o fato de o aluno ter reprovado em séries anteriores é determinante para o resultado final da nota de proficiência da escola, ou seja, a cada 1 p.p de alunos que já reprovaram, a nota da escola reduz em 86,36 pontos. Esta variável é importantíssima, pois demonstra como background escolar é determinante na vida estudantil dos alunos.

No que tange as características dos professores, as variáveis Msal_ate3 e Prof_exp_5 $5^{\underline{a}}$ foram significativas, contudo, influenciam negativamente a nota da escola, isto é, a cada 1 p.p. de aumento de professores nessa faixa salarial, a nota cai em 11,66 pontos. Resultados parecidos foram encontrados por Silveira (2014), indicando o baixo salário dos professores não torna a carreira atrativa além de não incentivar a especialização dos mesmos. Além disso, outra explicação para isso é a imediata saída do recém-formado da universidade para atuar como doente na sala de aula. Ademias, vale destaca que o curso de matemática é um dos menos concorridos nas universidades brasileiras, e isso leva vários alunos que já apresentavam dificuldade com a disciplina à época da escola a disputar uma vaga, e ocasionalmente irão ministrá-la nas séries iniciais fazendo assim um ciclo vicioso.

Revista de Estudos Sociais | Ano 2017, Pag. 13 
Com relação à infraestrutura da escola, somente a variável Esc_biblioteca foi significativa estatisticamente, mostrando que escolas com bibliotecas aumentam a proficiência dos seus alunos na disciplina de matemática, resultado semelhante ao encontrado por Menezes-Filho (2007). Ademais, conforme salienta Mayrink (1991), a biblioteca constitui um fator muito importante na formação do indivíduo, pois estimula a criatividade, a comunicação, além de ser um instrumento de desenvolvimento do currículo, fomentando a leitura e a formação de uma atividade científica, desde que utilizada de forma correta e por profissionais capacitados.

\section{CONSIDERAÇÕES FINAIS}

O investimento em educação é primordial para a construção de uma nação rica, tanto em termos econômicos quanto em termos culturais e principalmente sociais. Como é observado em vários países ao redor do mundo quando se investe em educação muitos problemas sociais são desfeitos, logicamente que não é apenas destinar o uma parte do PIB e tudo estará resolvido, é necessário alocar esses recursos, os quais são escassos, da maneira mais efetiva possível.

Diante disso, este estudo objetivou analisar o impacto do FUNDEB sobre o desempenho escolar do ensino médio da rede pública do estado do Amazonas, em especial no tocante ao nível de proficiência em matemática. O método a qual o presente estudo se valeu para tal análise foi o Diferenças em Diferenças.

Os resultados demonstraram que o FUNDEB não possui efeito sobre a proficiência em matemática das escolas públicas, o que demonstra que o fato de dispor de recursos não é determinante para melhorar o ensino. Algumas variáveis referentes às características dos alunos, dos professores e das escolas se mostraram estritamente sensíveis no desempenho dos alunos e consequentemente no desempenho das escolas. Variáveis que mensuravam a proporção de alunos do sexo masculino, alunos que já foram reprovados, alunos que possuem alguma atividade profissional e alunos que possuem pais com nível superior impactaram positivamente e negativamente no nível de proficiência médio das escolas.

Em contrapartida, a variável utilizada para controlar a cor ou raça, isto é, a proporção de alunos na cor preta, não se mostrou significante. Quanto às características dos professores e das escolas as variáveis que tratam da remuneração e escolas que possuem biblioteca respectivamente manifestaram impacto negativo e positivo. Algo marcante foi o fato de a escola possuir computador e internet, esta variável não apresentou nenhum impacto na nota final, é preciso adentrar mais neste efeito, pois grandes investimos estão sendo feitos com intuito de digitalizar as escolas.

Por fim, pelo que foi constatado o efeito do FUNDEB não foi eficiente para melhorar a educação. Tal fato cria uma discussão em torno de como estão sendo destinados esses recursos, os quais levam a crer que no modelo atual de destinação o ensino-aprendizagem da disciplina de matemática não está sendo favorecido.

\section{REFERÊNCIAS}

Revista de Estudos Sociais | Ano 2017, Pag. 14 
ATHEY, S; IMBENS, G. B. Identification and Inference in Nonlinear DifferenceIn-Differences Models. Technical Working Paper Series, Cambrige, n. 280, september, 2002.

BRASIL. Lei n. 11.494, de 20 de junho de 2007. Regulamenta o Fundo de Manutenção e Desenvolvimento da Educação Básica e de Valorização dos Profissionais da Educação - FUNDEB, de que trata o art. 60 do Ato das Disposições Constitucionais Transitórias; altera a Lei n. 10.195, de 14 de fevereiro de 2001; revoga dispositivos das Leis n. 9.424, de 24 de dezembro de 1996, 10.880, de 9 de junho de 2004, e 10.845, de 5 de março de 2004; e dá outras providências. Diário Oficial da União, Brasília - DF, 21 jun. 2007.

CPS /FGV - Centro de Políticas Sociais da Fundação Getúlio Vargas. Metodologia de Diferenças em Diferenças, Disponível em:

$<$ http://www.cps.fgv.br/cps/pesquisas/Politicas sociais alunos/2010/20100512/ PDF/BES Diferen\%C3\%A7asemDiferen\%C3\%A7as.pdf>

DOURADO, L. F. Elaboração de Políticas e Estratégias para a Prevenção do Fracasso Escolar, Ministério da Educação, Secretaria de Educação Infantil e Fundamental, Brasília, 2005.

FERNANDES, D. Brasil avança em conhecimento básico de matemática, mas continua atrás em ranking. BBC Brasil, Paris, 10 fev. 2016. Sítio: http://www.bbc.com/portuguese/noticias/2016/02/160209 ocde alunos baixa performance pai df. Data de acesso: 29/11/2016.

FOGUEL, M. N. Modelo de Resultados Potenciais. In: Naercio Menezes Filho (Org.). Avaliação Econômica de Projetos Sociais, 1ำed., São Paulo - SP, Dinâmica Gráfica e Editora, p. 35-48, 2012.

FRANCO, A. M. P. Os Determinantes da Qualidade da Educação no Brasil. Tese (Dourotado em Economia). Faculdade de Economia, Administração e Contabilidade, Universidade de São Paulo, São Paulo, 154 p, 2008.

GLEWWE, P.; KREMER, M. Schools, Teachers and Educacional Outcomes in Developing Countries. In: Haushek E. A. (ed.). Handbook of the Economics of Education. Amsterdam: North Holland, p. 943-1.017, 2006.

HANUSHEK, E. A. Teacher Quality. In: IZUME, L. T; EVERS, W. M. (ed). Teacher Quality. Stanford: Hoover Institution Press, p. 1-12, 2002.

IBGE - Instituto Brasileiro de Geografia e Estatística. Síntese de indicadores sociais: uma análise das condições de vida da população brasileira, Rio de Janeiro, 2013.

INEP - Instituto Nacional de Estudos e Pesquisas Educacionais Anísio Teixeira. Sistema de Avaliação da Educação Básica. Manual do Usuário. Brasília, DF, 2011a. 
. Resultados 2011. Brasília, DF, 2011b.

2011c.

Sinopses Estatísticas da Educação Básica. Brasília, DF. 2005,

. Censo Escolar. Brasília, DF.

Prova Brasil 2013. Brasília, DF.

Resultados do Índice de Desenvolvimento da Educação Básica 2005-2015. Resumo Técnico, Diretoria de Estatísticas Educacionais, Brasília, DF, 2016.

LEONARDO, P. P; MENESTRINA, T. C; MIARKA, R. A importância do ensino da matemática na educação infantil. In: I Simpósio Educação Matemática em Debate, SIMPEMAD, Joinville, SC, 2014.

MACHADO, A. F.; et al. Qualidade do Ensino em Matemática: Determinantes do Desempenho de Alunos em Escolas Públicas Estaduais Mineiras. Revista Economia, Brasília (DF), v. 9, n.1, p. 23-45, jan./abr., 2008.

MAYRINK, P. T. Diretrizes para a Formação de Coleções de Bibliotecas Escolares. In: Congresso Brasileiro de Biblioteconomia e Documentação, 16, 1991, Salvador. Anais Salvador: Associação Profissional nos Bibliotecários do Estado da Bahia, 2 v, v1, p.304-314, 1991.

MENEZES-FILHO, N. Os determinantes do desempenho escolar do Brasil. Instituto Futuro Brasil, IBEMEC-SP e FEA-USP, São Paulo, 2007. Insper, 2011.

Valorizar o professor ou o mérito? Instituto de ensino e Pesquisa -

MENEZES-FILHO, N.; PAZELLO, E. Do teachers' wages matter for proficiency? Evidence from a funding reform in Brazil. Economics of Education Review, $v$. 26, p. 660-672, 2007.

MEC - Ministério da Educação e Cultura/Secretaria de Educação Fundamental. Parâmetros Curriculares Nacionais. Brasília: MEC/SEF, 1997.

PNAD- Pesquisa Nacional por Amostra de Domícilios: Síntese de indicadores 2014/IBGE, Coordenação de Trabalho e Rendimento. Rio de Janeiro: IBGE, 2015.

RUBIN, D. Estimating Causal Effects of Treatments in Randomized and Nonrandomized Studies. Journal of Educational Psychology, v. 66, n. 5, p. 688$701,1974$.

SADOVSKY, P. Falta Fundamentação Didática no Ensino da Matemática. Nova

Revista de Estudos Sociais | Ano 2017, Pag. 16 
Escola. São Paulo, Ed. Abril, Jan./Fev. 2007.

SANTOS, Boaventura de Sousa. Um Discurso Sobre as Ciências. São Paulo: Cortez, 2006.

SEDEAM - Sistema de Avaliação do Desempenho Educacional do Amazonas. Revista Pedagógica com resultados do Sistema, Centro de Políticas Públicas e Avaliação da Educação (CAEd), Juiz de Fora, MG, 2011.

Revista Pedagógica com resultados do Sistema, Centro de Políticas Públicas e Avaliação da Educação (CAEd), Juiz de Fora, MG, 2015.

SILVEIRA, I. M. Avaliação do Efeito do FUNDEB sobre o desempenho dos alunos do ensino médio no Brasil. 2014. 66 p. Dissertação (Mestrado em Economia). Universidade Federal de Viçosa, Viçosa, 2014.

SCHULTZ, T. W.. O Capital Humano: investimentos em educação e pesquisa. Tradução de Marco Aurélio de Moura Matos. Rio de Janeiro: Zahar, 1973.

TOLEDO, L.F. Renda maior pesa mais no desempenho até dos estudantes das redes públicas; desigualdade cresce. Estadão, São Paulo, 11 set. 2016. Sítio: http://educacao.estadao.com.br/noticias/geral,renda-maior-pesa-nodesempenho-ate-dos-estudantes-das-redes-publicas, 10000075279. Data de acesso: 29/11/2016.

TREVISAN, Andrei Pittol; BELLEN, Hans Michael van. Avaliação de políticas públicas: uma revisão teórica de um campo em construção. Revista de Administração Púbica, Rio de Janeiro - RJ, v. 42, n. 3, p. 529-50, maio/jun. 2008 